Supporting Information for:

\title{
Structure and Dynamics of Poly(T) Single Strand DNA: Implications Toward CPD Formation
}

\author{
Andrew T. Johnson and Olaf Wiest* \\ *Department of Chemistry and Biochemistry, University of Notre Dame, \\ Notre Dame, Indiana 46556-5670 (USA) \\ owiest@nd.edu
}

C5-C5' Radial Distribution Functions of Distance

S2

Histograms of C5-C6-C6'C5' Dihedrals

S3

Snapshots From MD Trajectory

S4, S5 
Supplementary Information for: Johnson, A.T. \& Wiest, O.: Structure and Dynamics.. S2

C5: (1-2) to (4-5)

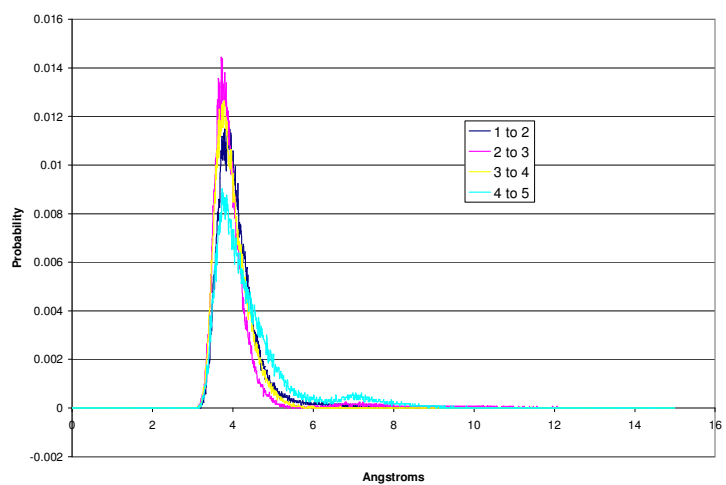

C5: (9-10) to (12-13)

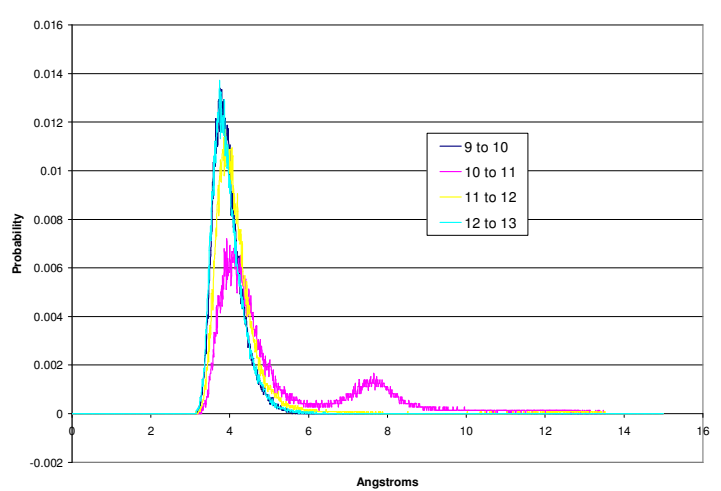

C5: (5-6) to (8-9)

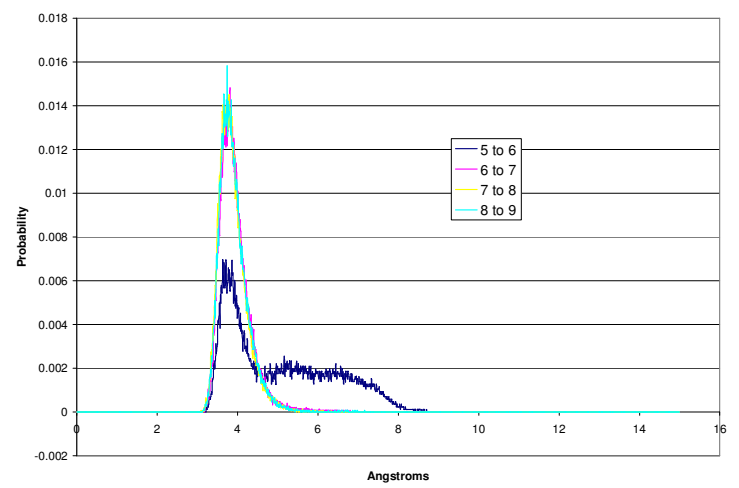

C5: (13-14) to (17-18)

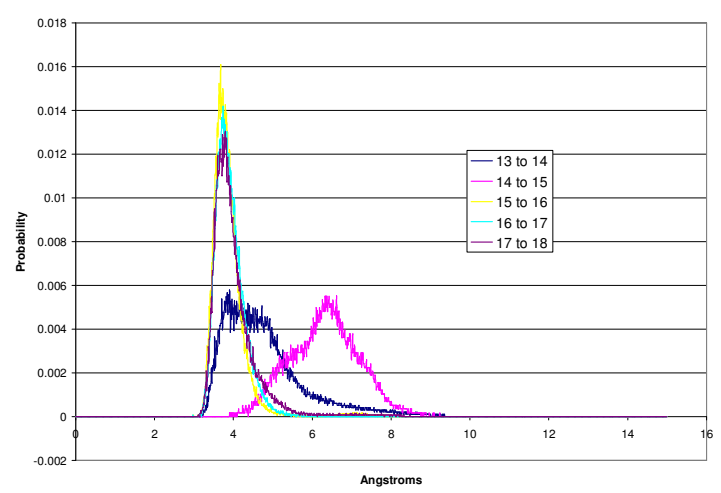

Figure S1: Radial distribution functions for C5-C5' distances from 50 ns MD simulation. Bases 1 and 18 are located at the 5' and 3' ends respectively. 
Supplementary Information for: Johnson, A.T. \& Wiest, O.: Structure and Dynamics.. S3
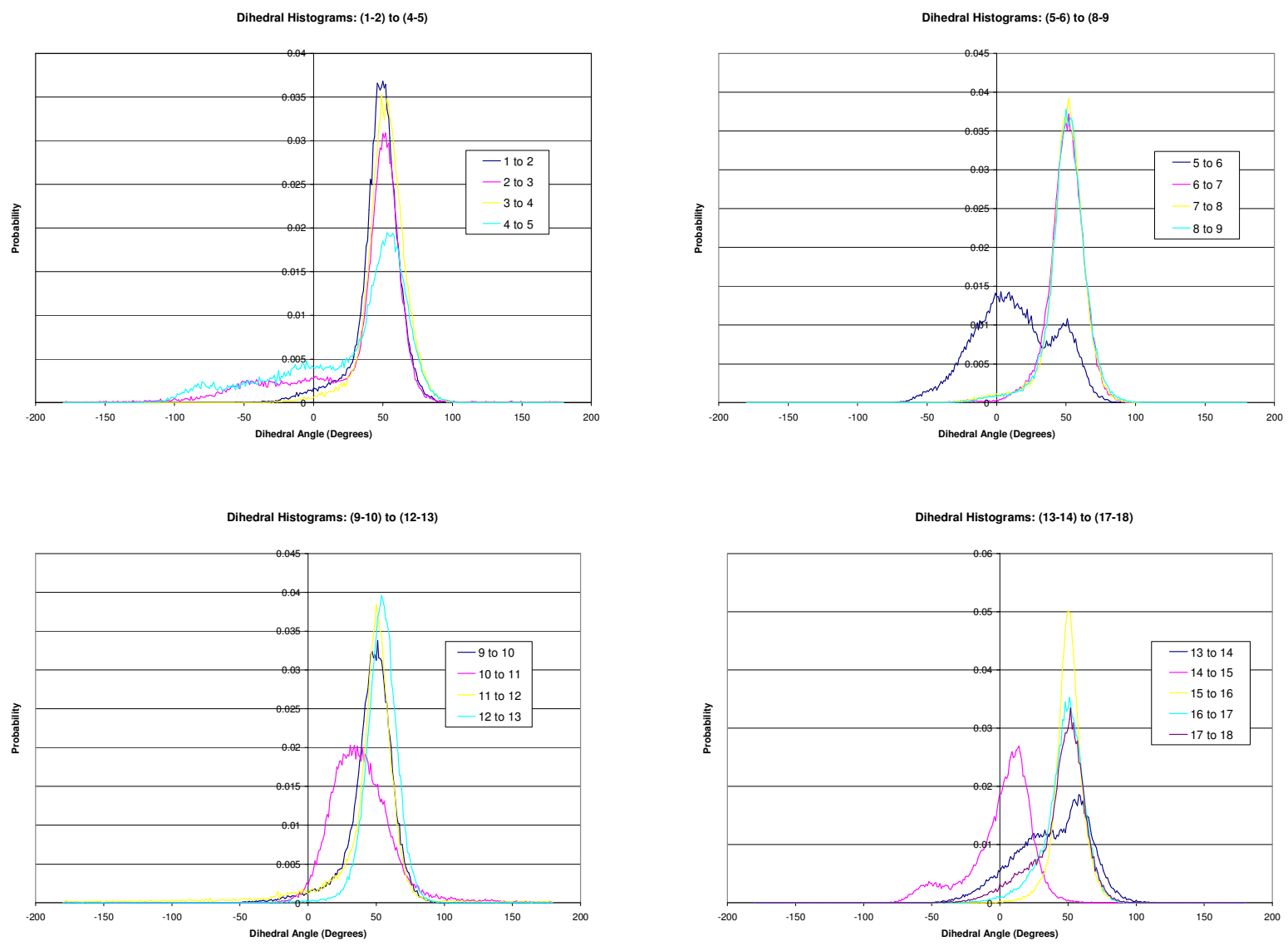

Figure S2: Histograms of C5-C6-C6'-C5' dihedral angles between individual base stacks for MD simulation. 
Supplementary Information for: Johnson, A.T. \& Wiest, O.: Structure and Dynamics.. S4
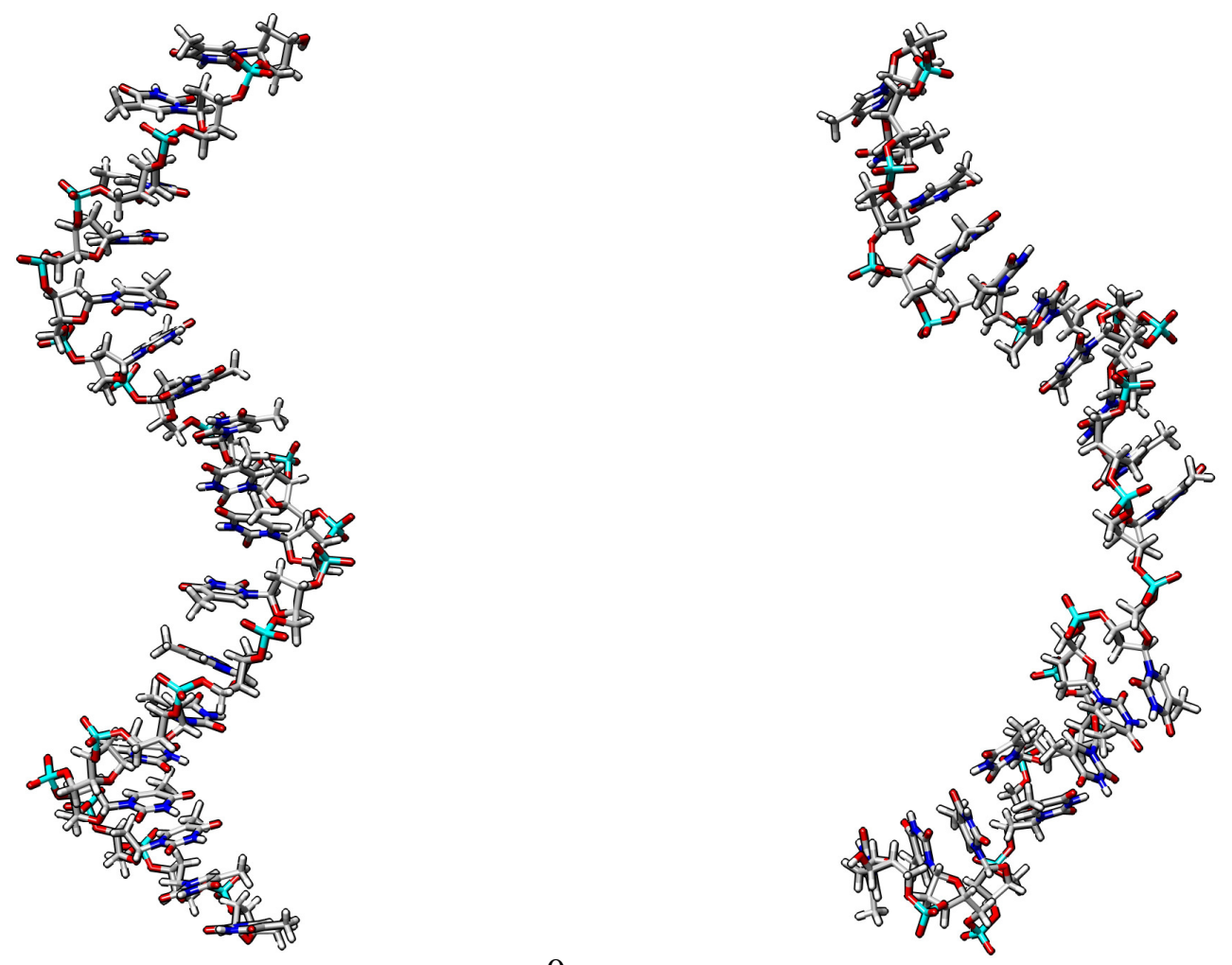

Ons

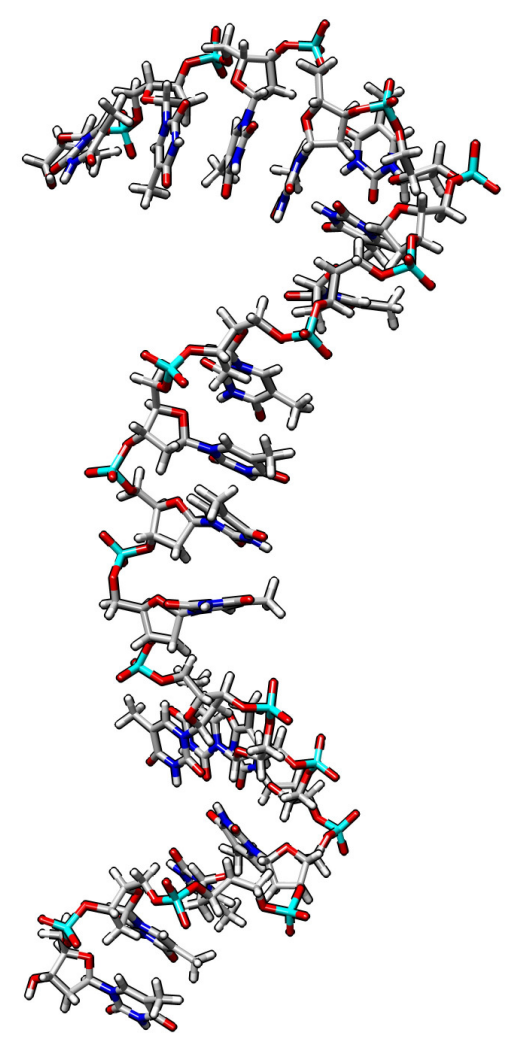

20ns

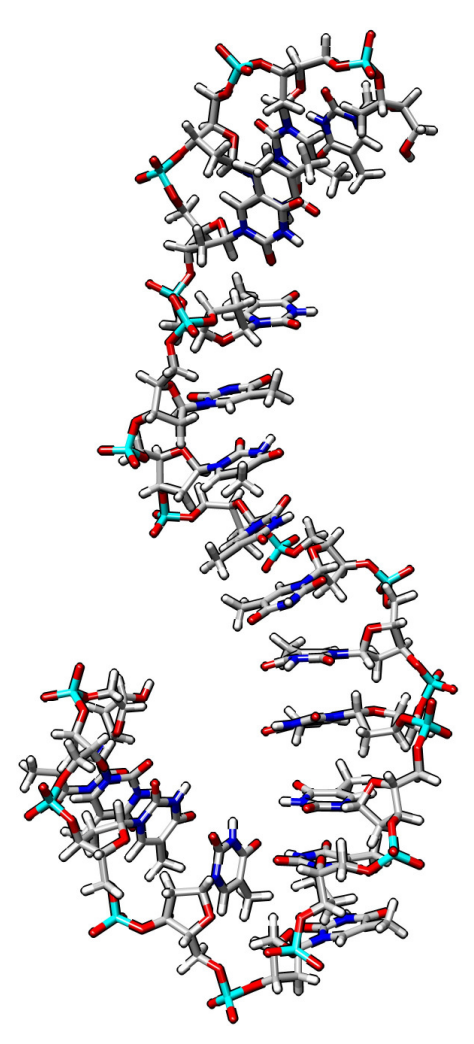


Supplementary Information for: Johnson, A.T. \& Wiest, O.: Structure and Dynamics.. S5

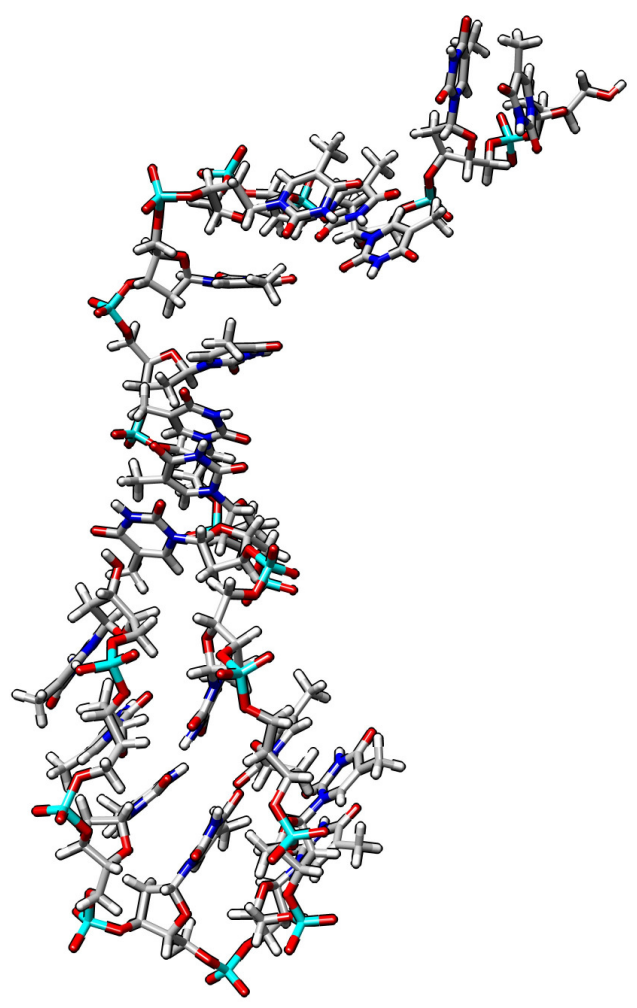

$40 \mathrm{~ns}$

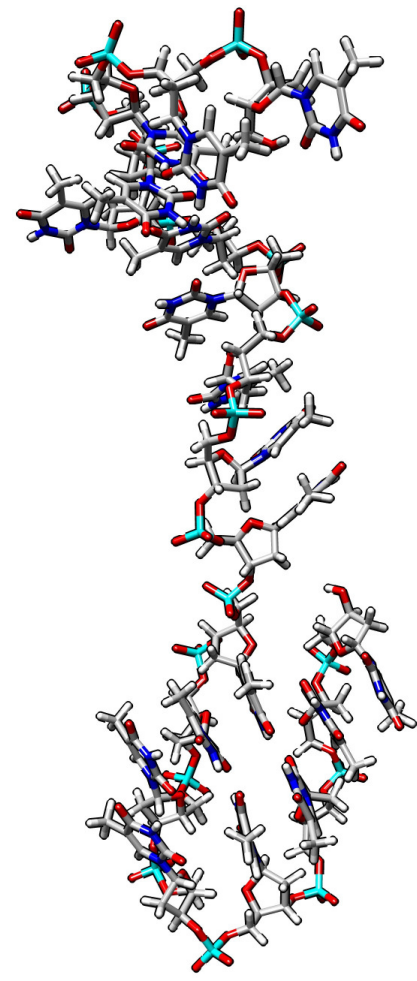

$50 \mathrm{~ns}$

Figure S3: Snapshots from $50 \mathrm{~ns}$ MD trajectory. Pdb files are available online at http://pubs.acs.org. 